\title{
Alternating Magnetic Field Forces for Satellite Formation Flying
}

\author{
Robert C. Youngquist ${ }^{\mathrm{a}}$, Mark A. Nurge ${ }^{\mathrm{a}, *}$, and Stanley O. Starr ${ }^{\mathrm{a}}$ \\ ${ }^{a}$ Mail-Stop NE-L5, National Aeronautics and Space Administration (NASA), Kennedy Space Center, FL 32899 \\ * Corresponding author. Tel.1-321-867-9938. E-mail address: Mark.A.Nurge@nasa.gov
}

\begin{abstract}
Selected future space missions, such as large aperture telescopes and multi-component interferometers, will require the precise positioning of a number of isolated satellites, yet many of the suggested approaches for providing satellites positioning forces have serious limitations. In this paper we propose a new approach, capable of providing both position and orientation forces, that resolves or alleviates many of these problems. We show that by using alternating fields and currents that finely-controlled forces can be induced on the satellites, which can be individually selected through frequency allocation. We also show, through analysis and experiment, that near field operation is feasible and can provide sufficient force and the necessary degrees of freedom to accurately position and orient small satellites relative to one another. In particular, the case of a telescope with a large number of free mirrors is developed to provide an example of the concept. We also discuss the far field extension of this concept.
\end{abstract}

Index Terms - Spacecraft formation flying, satellite propulsion, antenna forces, electromagnetic forces.

\section{INTRODUCTION}

ORBITING a large number of satellites in fixed formations will be critical to many future space missions, especially large scale interferometers, telescopes, antennas, and gravity wave detectors $[1,2]$. Consequently, extensive research has been devoted over the last 20 years to formation flying architectures, concentrating not only on the mission objective, but also on the technologies required to achieve a stable satellite formation. Several proposals have been suggested for determining the location of the satellites, but the more difficult problem is developing a system that can hold the satellites at those desired locations and orientations. The two most common "solutions" are to use micro-thrusters [3], though these require propellant and will eventually be depleted, or to choose orbital patterns that minimize relative perturbations [4,5], but for highly precise positioning this is not adequate. Neither of these approaches solves the problem for long duration missions such as a multi-element telescope where the mirrors must be located and oriented to a tolerance less than an optical wavelength.

NIAC has recognized this need and has funded three efforts exploring alternative methods to enable long term satellite formation flying. Briefly reviewing these:

1. Sedwick and Miller studied "Electromagnetic Formation Flight" $[6,7]$. In this approach superconducting magnets are placed on each satellite to provide short range attractive and repulsive forces. This approach is technically feasible and others have continued the work [8], but it adds significant mass and complexity to the satellites, requires reaction wheels for orientation control, and has limited range.

2. LaPointe studied "Formation Flying with Shepherd Satellites" [9]. In this concept a group of shepherd satellites circle and continually position the formation satellites using radiation pressure and electromagnetic gradient forces. This is interesting, but it transfers the problem to the shepherd satellites, which need their own positioning system.

3. Bae studied "A Contamination-Free Ultrahigh Precision Formation Flight Method Based on Intracavity Photon Thrusters and Tethers" [10]. This concept proposed the use of optical resonant cavities to enhance radiation pressure, pushing the satellites apart, and tethers to pull them back together. This is a high risk approach fraught with numerous technical issues, for example, aligning an optically resonant cavity at 10 meters is difficult on earth, but this method suggests doing it over kilometer distances with tethers strung between the satellites.

Ideally, a method is needed that can position and orient satellites relative to each other with the following attributes.

a. It should provide a wide dynamic range of forces in order to provide a deployment or formation rearrangement capability, yet also allow very fine position and orientation adjustments,

b. It should provide enough degrees of freedom that a large a number of satellites can be independently positioned and orientated with minimal cross-talk issues,

c. It should require minimal weight and power and not use propellants that can be exhausted,

d. It should be applicable to both short range, $(<100 \mathrm{~m})$ and long range ( $>100 \mathrm{~m})$ applications.

This paper proposes a method that meets most of these attributes. 


\section{Alternating MAGNETIC FIELD ForCES}

The proposed satellite formation flying approach is based on a well known effect, namely that the alternating magnetic field generated by modulating the current in one antenna or coil can interact with the synchronously modulated current in a second coil or antenna to produce a net force and net torque between the two [11]. Even though this is well known, what is novel is that this approach allows frequency allocation of forces, generating the necessary degrees of freedom to provide independent position and orientation forces for a large number of satellites. It also provides secondary benefits that include fine force control through phase and amplitude modulation, a wide dynamic range of forces, and both near and far field configurations. Most of this paper is devoted to near field considerations with Section $\mathrm{V}$ discussing the far field case.

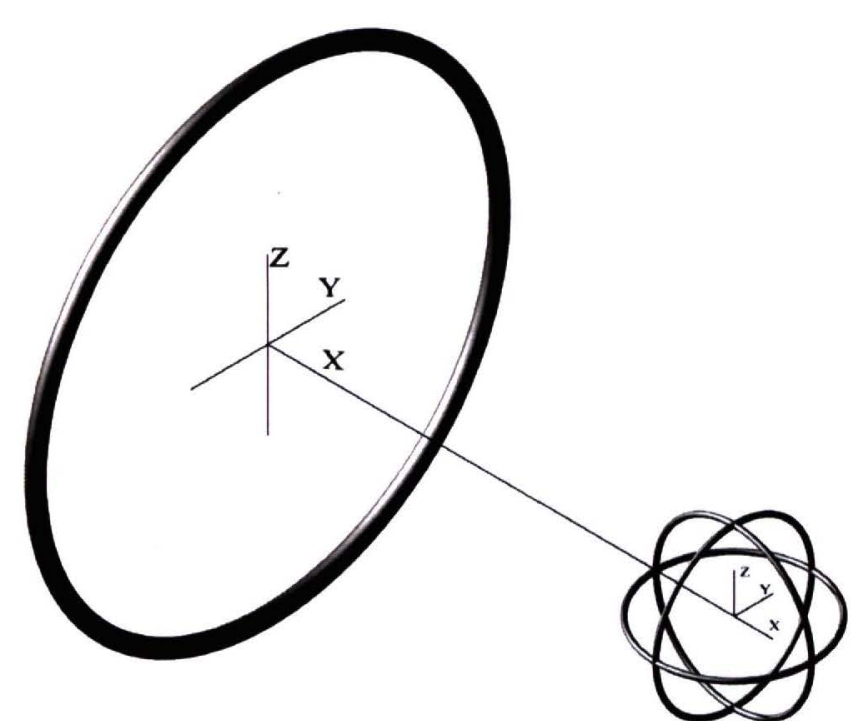

Figure 1. The large coil generates an oscillatory magnetic field at the smaller structure composed of three orthogonal coils. The three coils can be driven synchronously to yield an attractive or repulsive force along the $\mathrm{X}$ axis and two torque components about the $\mathrm{Z}$ and $\mathrm{Y}$ axes.

Start with the arrangement shown in Figure 1, where a large coil is located in the $\mathrm{Y} / \mathrm{Z}$ plane and a small structure composed of three identical, yet orthogonal, coils is located some distance from the large coil along the $\mathrm{X}$ axis. Assume the large coil is driven with a modulated current to produce a magnetic field throughout local space given by $\vec{B}(x, y, z) \cos (2 \pi f t)$. Also assume that the three smaller coils are driven with modulated currents given by $I_{x} \cos (2 \pi f t+\varphi), \quad I_{y} \cos (2 \pi f t+\varphi)$, and $I_{z} \cos (2 \pi f t+\varphi)$, i.e., all three smaller coils are driven at the same frequency and phase, but with different amplitudes. Note that the subscript on the current corresponds to the axis of the respective coil, e.g. $I_{x}$ is the current in the small coil in the $\mathrm{Y} / \mathrm{Z}$ plane.

Now assume that the three-coil structure is small enough that the force and torque on it can be approximated by the well-known dipole expressions [12]

$$
\begin{aligned}
\vec{F} & =\nabla(\vec{m} \cdot \vec{B}) \\
\vec{\tau} & =\vec{m} \times \vec{B}
\end{aligned}
$$

where $\vec{m}$ is the dipole moment. The dipole moment, $\vec{m}$, for the three coil structure is given by

$$
\vec{m}=\left(I_{x} \hat{x}+I_{y} \hat{y}+I_{z} \hat{z}\right) N A \cos (2 \pi f t+\varphi)
$$

where $N$ is the number of loops in each coil and $A$ is the area of each coil. Since the current in each coil can be independently controlled the dipole moment can point in any desired direction. Consequently, a portion of the dipole moment can be aligned with the magnetic field, along the $\mathrm{X}$ axis in Figure 1. Since along the $\mathrm{X}$ axis the magnetic field has only an $\hat{x}$ component the force on the three-coil structure is given by

$$
F_{x}=\frac{\partial}{\partial x}\left(I_{x} N A B(x) \cos (2 \pi f t) \cos (2 \pi f t+\varphi)\right)
$$

Note that there is a product of cosines in this equation which can be expanded to yield a constant term and a term that oscillates at a frequency of $2 f$. The oscillatory force yields a displacement with a magnitude proportional to $1 /(4 \pi f)^{2}$, e.g. a factor of about $10^{-8}$ for a $1 \mathrm{kHz}$ oscillation and can be dropped in most cases. So the net force is determined by the constant term yielding

$$
F_{x}=\frac{\partial}{\partial x}(B(x)) I_{x} N A \cos (\varphi) / 2 .
$$

Similarly, again referring to the situation shown in Figure 1, nonzero currents, $I_{y}$ or $I_{z}$, yield magnet dipole components that are orthogonal to the magnetic field and produce torque. Following the same argument as for the force and assuming that oscillatory torques can be ignored, the net torque is given by

$$
\vec{\tau}=\left(I_{z} \hat{y}-I_{y} \hat{z}\right) B N A \cos (\varphi) / 2 .
$$

So by controlling the currents in the three coils of the smaller structure one force component and two torque components can be varied, allowing partial positioning and orientation of these two entities relative to each other. (It should be recalled that for any force or torque felt by the smaller structure there is an opposite force or torque felt by the larger structure).

However the goal is to provide full position and orientation control of the smaller three-coil entity relative to the large coil. To achieve that additional large coils are required. Consider the idealistic situation shown in Figure 2 where three large coils are located in each of the three orthogonal planes and aligned with the three-coil structure 
along each of its axes. Let the current in the " $X$ " coil be modulated at frequency, $f_{X}$, the current in coil "Y" be modulated at a different frequency, $f_{Y}$, and coil " $Z$ ", modulated at a third frequency, $f_{Z}$. If we chose these three frequencies to be sufficiently different then their cross term induced forces and torques can be ignored and the analysis given above for the single large coil can be repeated for each of the two new large coils. Doing this, and driving the smaller coils with currents at these new frequencies, but with a chosen phase difference, yields a total force on the small three-coil structure given by

$$
\begin{aligned}
& \vec{F}=\frac{\partial}{\partial x}\left(B_{X}(x)\right) I_{x} \cos \left(\varphi_{x}\right) N A / 2 \hat{x} \\
& +\frac{\partial}{\partial y}\left(B_{Y}(y)\right) I_{y} \cos \left(\varphi_{y}\right) N A / 2 \hat{y}+\frac{\partial}{\partial z}\left(B_{Z}(z)\right) I_{z} \cos \left(\varphi_{z}\right) N A / 2 \hat{z}
\end{aligned}
$$

Since the current magnitude and phases can be chosen this demonstrates that in such a configuration that complete control of the force on the small three-coil structure is possible. Similarly, complete control of the torque is also now achievable.

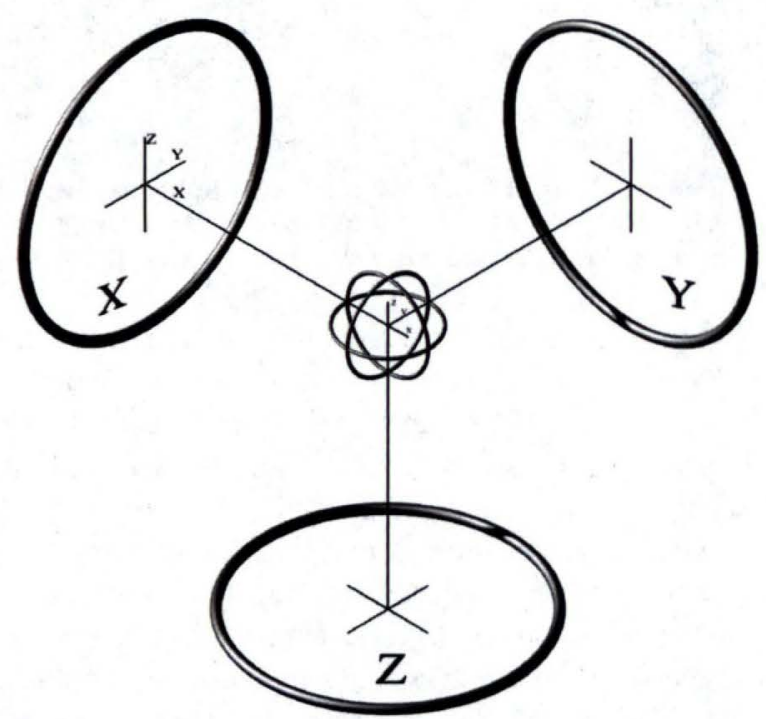

Figure 2. By locating three large coils around the smaller three-coil structure full position and orientation control is possible.

In a more realistic scenario the large coils are not necessarily orthogonal, nor aligned directly with the smaller three-coil structure. Yet, all that is necessary for this approach to be feasible is that the magnetic fields generated by the large coils be vectorially independent of each other over the volume of space where the small three-coil structure is located. Figure 3 shows a more general configuration where we will now refer to the large coils as the drive coils and assume that they are locked together structurally as part of the primary structure of a satellite formation. The smaller three-coil structures will now be referred to as satellites and the goal is to be able to position and orient a large number of these satellites relative to the drive coils.

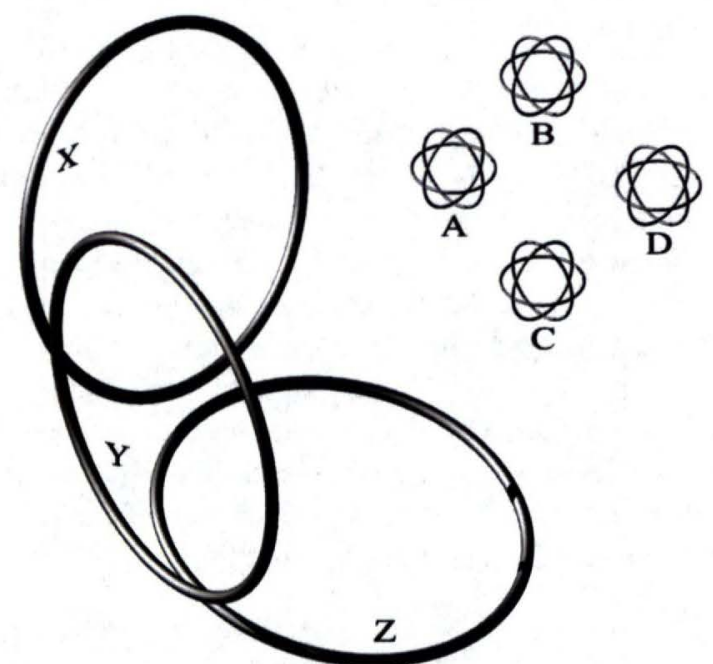

Figure 3. A more general drive coil/satellite configuration can provide full position and orientation control of a number of satellites.

It has been shown that satellite A's position and orientation can be controlled by driving the three large coils with three different frequencies, $f_{X, A}, f_{Y, A}$, and $f_{Z, A}$, that are also the frequencies used in satellite A's coils. Similarly, using the three frequencies, $f_{X, B}, f_{Y, B}$, and $f_{Z, B}$, satellite B's position and orientation can be controlled. Now, the frequencies used in satellite A and in satellite B do not have to be different, but if they are the same then these two satellites will affect each other's position and orientation and the feedback and control problem becomes much more complicated (as an example of this see [7] where the control in 2-D of constant, i.e. nonoscillating, magnets is developed). Consequently, when fine control of the position and orientation of the satellites is desired it is preferable to allocate three different frequencies to each satellite.

This raises the question of how many frequencies are available, since this determines the number of satellites that can be independently controlled. The exact answer to this question depends on a number of application specific parameters, but a general result can be obtained by noting that each satellite vibrates due to the beating between its current frequencies and the magnetic fields generated by the large coils at other frequencies. These vibration forces, $F_{V}$, are of the same size as the control forces, $F_{C}$, given above and can be expressed as

$$
F_{V}=F_{C} \cos \left(2 \pi\left(f_{1}-f_{2}\right) t\right)
$$

where $f_{1}$ and $f_{2}$ are two different, but adjacent frequencies. Now, let $d$ be the maximum displacement allowed for one of the satellites and let $m$ be the mass of a satellite. Solving for this maximum displacement by integrating the force equation twice yields 


$$
d>\left(F_{C} / m\right)\left(1 /\left(2 \pi\left(f_{1}-f_{2}\right)\right)^{2}\right) \text {. }
$$

So, for example, if the control force is $10^{-4}$ Newtons, the satellite mass is $30 \mathrm{~kg}$, and the maximum displacement is $1 / 20^{\text {th }}$ of an optical wavelength, i.e. 0.03 microns, then the frequency difference is $1.7 \mathrm{Hertz}$. So if the large coils are configured to operate in the low kilohertz region and have a few hundred Hertz bandwidth then under these conservative assumptions the position and orientation of about 100 satellites can be controlled. There are other important design issues, such as choosing the appropriate large and small coil parameters so that maximum forces can be achieved with minimal power and weight. But these are application specific and will be left to Section IV where an example is developed to indicate how this design might proceed.

\section{EXPERIMENTAL VERIFICATIONS}

Two experiments were performed to show that oscillating magnetic fields and currents can generate predictable and significant, phase-controlled forces and torques. It should be mentioned that these experiments demonstrate no new physics, indeed similar experiments were done nearly 100 years ago [11], but they do demonstrate the degree to which torque and force can be finely controlled, the magnitude of forces that can be developed with simple coils and small currents, and ease with which such systems can be modeled.

In both experiments we use relatively large drive coils to develop a magnetic field in a volume of space where we locate a smaller pick-up coil acting as our satellite. The drive coils are $0.098 \mathrm{~m}$ in radius, $0.014 \mathrm{~m}$ in length, and have 100 wraps of 24 AWG magnet wire (total wire length is $62 \mathrm{~m}$ ) on a plastic mandrel. So the calculated resistance of the coil is 5.2 $\mathrm{Ohms}$ and the calculated inductance is given by $\left(\mu_{0} /(4 \pi)\right)\left(l^{2} / b\right) K$, where $l$ is the length of wire, $b$ is the coil length, and $K$ is a correction factor equal to $0.16[13,14]$, i.e. $4.4 \mathrm{mH}$. The measured resistance and inductance were 5.3 Ohms and $4.3 \mathrm{mH}$, close to the calculated values. The magnetic field along the axis of a flat coil is given by

$$
\vec{B}=\frac{\mu_{0} N I a^{2}}{2\left(a^{2}+x^{2}\right)^{3 / 2}} \vec{x}
$$

using the coordinate system shown in Figure 1 where $a$ is the drive coil radius, $N$ is the number of wraps, and $I$ is the current (positive in the counter-clockwise direction). For example, at the center of the coil, ( $x=0$ in Eqn. 4) and flowing $1 \mathrm{Amp}$ of current the magnetic field from this equation is $0.64 \mathrm{mTesla}$. The measured field was $0.75 \mathrm{mTesla}$ $+/-0.01 \mathrm{mTesla}$, somewhat higher than predicted due to the small extension of the actual coil versus the flat coil approximation.

In the first experiment we placed the two large drive coils seven inches apart and hung a small coil between them as shown in Figure 4. This generates a symmetric magnetic field allowing torque to be studied without significant translational force effects. The large drive coils were driven in series sinusoidally at $1 \mathrm{kHz}$ with $117 \mathrm{~mA}$ (peak) current, producing a peak magnetic field of $0.078 \mathrm{mTesla}$ in the center region between the coils along their axis. Note that the large coils now have a small mutual inductance causing their total inductance to increase to $8.9 \mathrm{mH}$. Also, eddy current generation in the small coil causes additional energy loss resulting in a total resistance for the two drive coils of 12.3 Ohms.

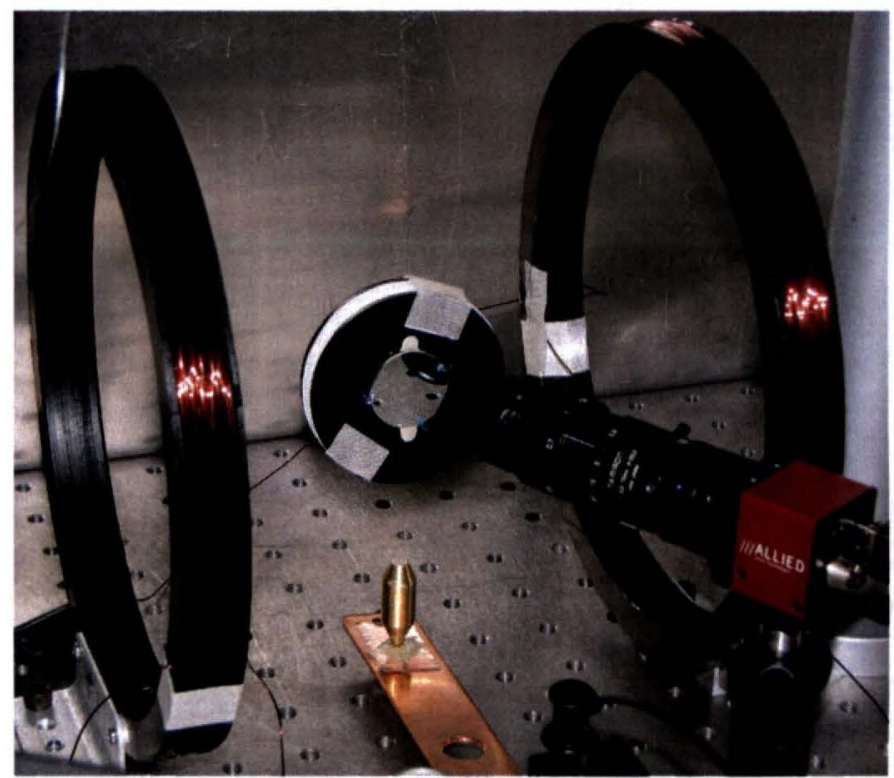

Figure 4. A small coil was suspended between two large drive coils to demonstrate alternating magnetic field induced torque.

The small coil was constructed by placing 100.5 wraps of 30 AWG wire onto a $9.2 \mathrm{~mm}$ wide plastic mirror mount. The coil diameter was 0.057 meters, yielding 18 meters of wire and a predicted resistance of $6.1 \mathrm{Ohm}$. It was suspended between the two drive coils, as shown in Figure 4, using a 0.25 meter length of steel wire $0.2 \mathrm{~mm}$ in diameter. An extended length of the steel wire was connected to a brass weight which hung in a pool of silver oil on a copper beam acting as the return path for the current running through the hanging coil. The total resistance of this coil and steel wire arrangement was $10.2 \mathrm{Ohms}$. The small coil has a calculated inductance of 0.99 $\mathrm{mH}$ and at $1 \mathrm{kHz}$ the measured inductance was $0.95 \mathrm{mH}$. The area of the small coil is $0.0026 \mathrm{~m}^{2}$ and the peak $1 \mathrm{kHz}$ modulated current placed into it was $32 \mathrm{~mA}$, so the peak dipole moment is $0.0084 \mathrm{~A}-\mathrm{m}^{2}$. Consequently, the expected torque from Eqn. 3 above is $3.3 \times 10^{-7} \cos \varphi \mathrm{N}-\mathrm{m}$ which will cause the mirror to turn against the restoring torque created by the steel wire.

The restoring torque of a steel wire is given by its torsion which can be calculated using

$$
\tau=\frac{\pi d^{4}}{32 l} G \theta
$$


where $d$ is the wire diameter, $l$ is the wire length, $G=80$ GPascals, the sheer modulus for steel, and $\theta$ is the angular twist in radians. For the case described this yields a restoring torque of $5 \times 10^{-5} \theta$, which agrees with a measured value of $4.8 \times 10^{-5} \theta$ obtained using the oscillation period of a torsion balance. Combining these two torque expressions yields a predicted angular extent for the mirror of $0.0068 \cos \varphi$, where $\varphi$ is the phase angle between the current oscillations. The phase difference, $\phi$, between the applied voltages is shifted by 0.82 radians from the applied current phase angle due to the impedance difference between the drive coils and the mirror coil, so the angular extent prediction in radians when monitoring voltage is $0.0068 \cos (\phi-0.82)$.

Referring back to Figure 4, a small camera is used to look into the mirror at a target composed of black and white stripes. Using an image processing algorithm the edge of this target can be tracked to accurately provide the angular motion of the mirror. The phase of the $1 \mathrm{kHz}$ voltages applied to the drive and mirror coil was varied linearly by $2 \pi$ radians over a one hour period, reading the mirror angle about 3 times a second. The result is shown in Figure 5 along with the theoretical prediction showing reasonable agreement. The triangular black sections correspond to the mirror oscillating after abrupt changes to the drive voltages. This result demonstrates that fine control of orientation can be achieved by modulating the phase between two coils being driven sinusoidally.

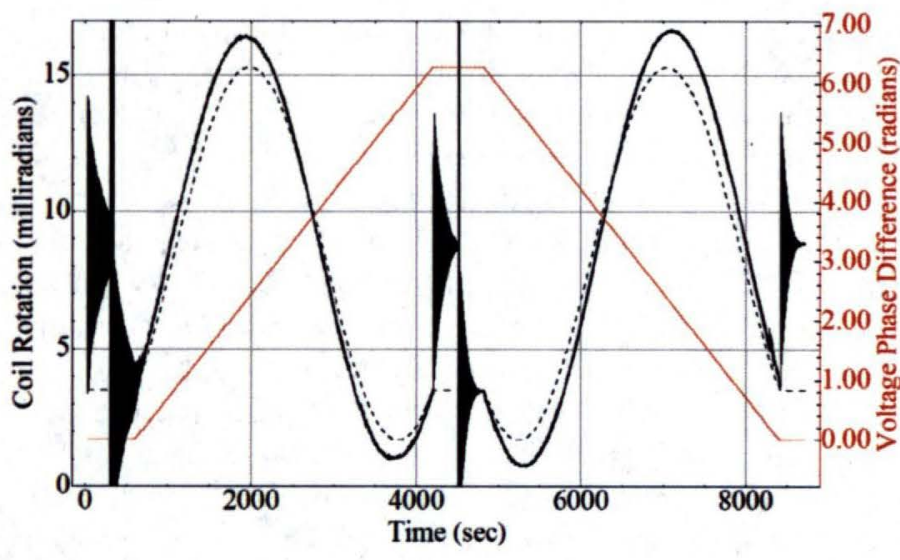

Figure 5. This plot shows the rotation caused by the torque generated by an oscillating magnetic field interacting with a synchronously oscillating coil current. The red plot shows how the phase difference between the coil voltages was varied in time and the black plots show the theoretical prediction (dashed line) and the experimental results (over 25000 data points). The dark triangles correspond to decaying mirror oscillations induced by sudden voltage changes.

In the second experiment we constructed a torsion balance, as shown in Figure 6, where the satellite coil is now fixed to a beam hanging from a thin coaxial cable. A large drive coil generates an oscillating magnetic field that decays along its axis producing a magnetic field gradient that can interact with the modulated current flowing through the small coil, resulting in a phase dependent net force. The large drive coil and the small coil have the same physical dimensions as the ones used in the torque experiment, but configuration differences and mutual inductance have altered the electrical specifications. At one kilohertz the large coil has $5.8 \mathrm{mH}$ inductance and $9.3 \mathrm{Ohms}$ resistance and the small coil has .97 $\mathrm{mH}$ inductance and $7.5 \mathrm{Ohms}$ resistance. The drive coil was driven with $290 \mathrm{mAmps}$ of peak current and the small coil with $350 \mathrm{mAmps}$ of peak current. The small coil was located approximately one radius of the large coil away from the large coil along its axis as shown in Figure 6. At this location the magnetic field gradient is $1.0 \mathrm{mT} / \mathrm{meter}$ (see Eqn. 6). The area of the small coil is $0.0026 \mathrm{~m}^{2}$ so its peak dipole moment is $0.091 \mathrm{~A}-\mathrm{m}^{2}$ and the corresponding peak force is, see Equation 2, 46 microNewtons. The moment arm is 0.27 meters long so the torque is $1.2 \times 10^{-5} \mathrm{~N}-\mathrm{m}$.

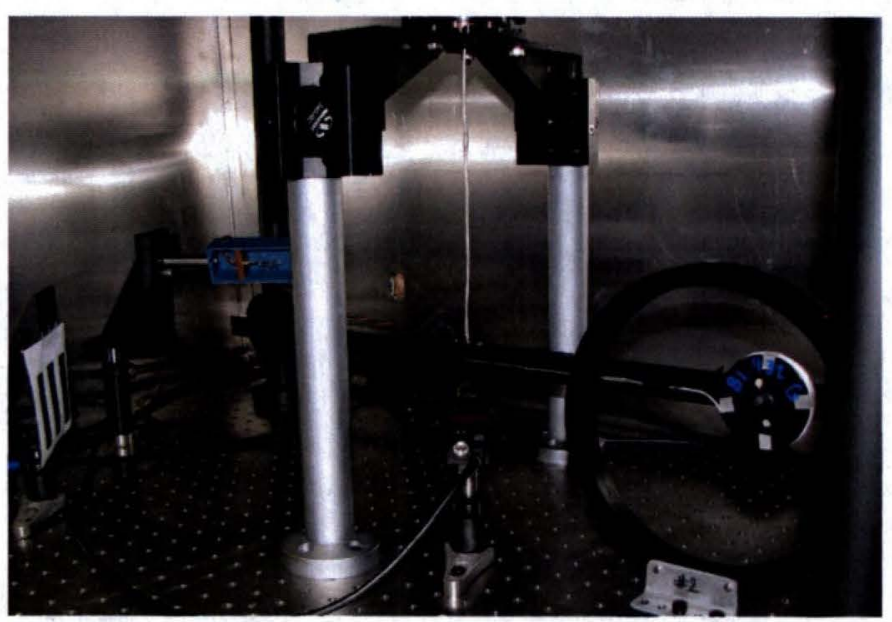

Figure 6. A torsion balance was used to measure the phase variations in force between a large drive coil and a small satellite coil.

The restoring torque of the co-axial cable cannot be easily calculated, but it can be measured by recalling that the spring constant of this cable is given by $I(2 \pi / T)^{2}$ where $I$ is the moment of inertia of the torsion balance and $T$ is the oscillation period. The moment of inertia is found to be $I=.025 \mathrm{~kg}-\mathrm{m}^{2}$ and the period is 11.9 seconds yielding a spring constant of $7.0 \mathrm{mN}-\mathrm{m} / \mathrm{rad}$, roughly 100 times larger than for the thin steel wire used in the torque experiment, yielding a 1.7 mrad peak deflection. The coil voltages are shifted in phase with respect to the applied currents due to the impedance difference between the drive coils and the mirror coil. Taking this into account the angular extent prediction in radians when monitoring voltage is $0.0017 \cos (\phi-0.44)$.

Again, a camera was used to track rotation by looking at the image of a black and white pattern in a mirror attached to the torsion balance, see Figure 6 . The phase difference in voltage between the drive coil and torsion balance coil was slowly varied from 0 through $2 \pi$ radians with the results shown in Figure 7 alongside the theoretical curve. The amplitude discrepancy is likely due to the use of Equation 1 to derive the force. This equation assumes the magnetic field derivative is uniform across the dipole, which is an approximation in the present case. Taking this into account the agreement is reasonable. 


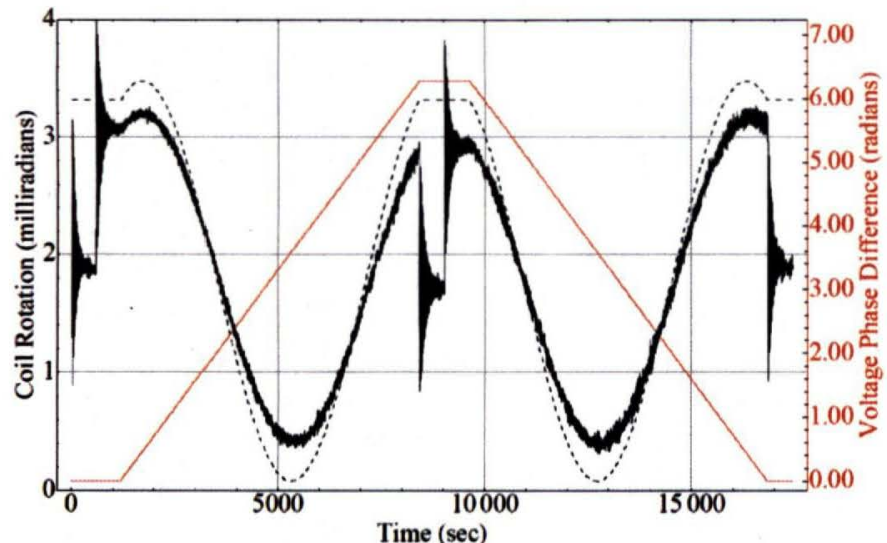

Figure 7. This plot shows the rotation of a torsion balance caused by the torque generated by an oscillating magnetic field interacting with a synchronously oscillating coil current. The red plot shows how the phase difference between the coil voltages was varied in time and the black plots show the theoretical prediction (dashed line) and the experimental results (over 50000 data points). The dark triangles correspond to decaying mirror oscillations induced by sudden voltage changes. The data has not been averaged, but a small linear rotation angle drift has been removed.

These two experiments demonstrate not only the expected result that synchronous alternating magnetic fields and currents produce net forces, but that these forces can be finely controlled through phase modulation and that substantial torques and forces can be achieved with modest coils and currents.

\section{AN EXAMPLE; A NeXT GeNERATION TELESCOPE}

One application of this satellite positioning and orientation concept would be the creation of a next generation large aperture telescope. The Hubble telescope has a 2.4 meter diameter collecting aperture [15], the James Webb telescope has a 6.5 meter aperture [16], and in this section we extend this progression and present a possible 20 meter aperture telescope configuration. Both the Hubble and James Webb Telescopes use large primary mirrors to collect light and reflect it to a smaller secondary mirror which then directs the light to a telescope sub-module containing additional optics, focal plane arrays, and processing electronics. The Hubble telescope has one large primary mirror element while the James Webb telescope's primary mirror is composed of 18 smaller hexagonal mirrors, each about 1.3 meters in diameter and weighing about 20 kilograms.

We propose to use 60 mirrors of the same size, shape, and weight as in the James Webb Telescope, but to attach three orthogonal coils to each of these mirrors so that they can be free floating. A system sketch is shown in Figure 8 with four of the 60 primary mirror elements shown. Three large drive coils are used to provide the oscillating magnetic fields, as described above in Section II, from which the small mirrors can generate orientational and positional forces. A free floating secondary mirror is located on the system axis to direct the light from the primary mirror elements back to the sub-module. A possible arrangement for the 60 primary mirror elements is shown in Figure 9 yielding a primary mirror with three times the collecting area and three times the diameter of the James Webb telescope.

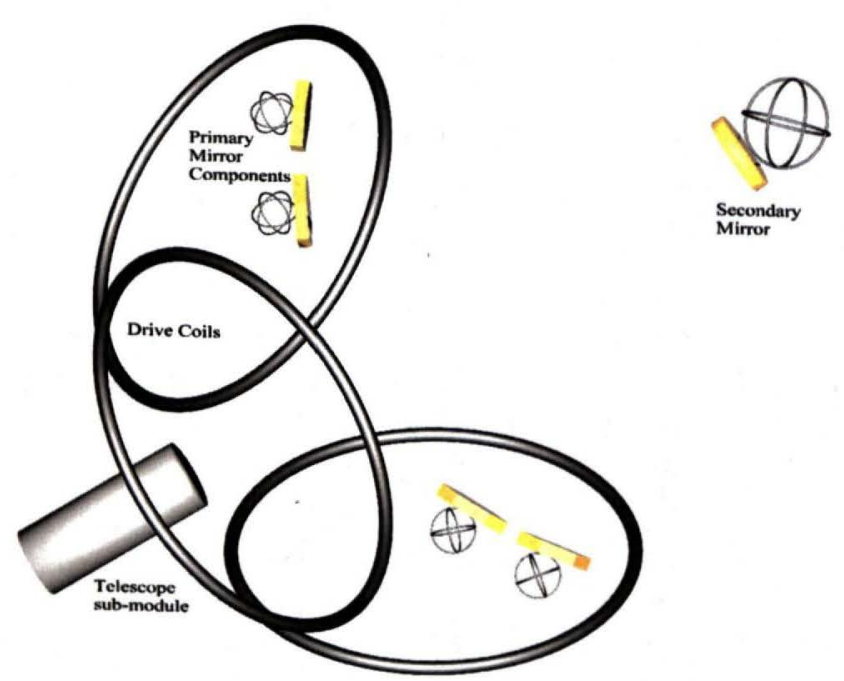

Figure 8 . A possible future telescope utlizing alternating magnetic field forces would use three large drive coils to allow a large number of satellite mirrors to be positioned and oriented accurately. In this conceptual image only four out of the 60 mirrors proposed for the primary reflector are shown and they are not to scale with the drive coils.

The coils on the primary mirror elements will require electronics, batteries or capacitors, and a power generation/collection system, so we estimate each mirror's total weight to be about 30 kilograms. The maximum force required to position these mirrors will occur during rotation of the telescope, i.e. a change in pointing direction, where we assume that 4 hours are needed to move distances up to 40 meters. Moving in a straight line this would require a force of 23 micro-Newtons for 2 hours and then an opposing and equal force for 2 hours to stop the mirror. Since the mirrors will not follow a straight path we will assume that a desired maximum force on the mirrors is $\mathbf{5 0}$ micro-Newtons.

Once the telescope is oriented and the mirror elements are in position only small forces are required to maintain the mirror element configuration. We assume the telescope under discussion, like the James Webb telescope, will be located at the Earth-Sun L2 point where gravitational gradients due to the sun-moon-earth are small and where a solar shield will likely be used for cooling and will minimize radiation pressure effects. Consequently, we believe the most significant forces causing the primary mirror elements to drift away from their optimal positions is gravitational attraction to the sub-module. Assuming this element weighs 4000 kilograms and is 10 meters from the nearest 30 kilogram mirror elements, the gravitational force is 80 nano-Newtons. (The gravitational force between neighboring mirror elements is approximately 15 nano-Newtons.) 


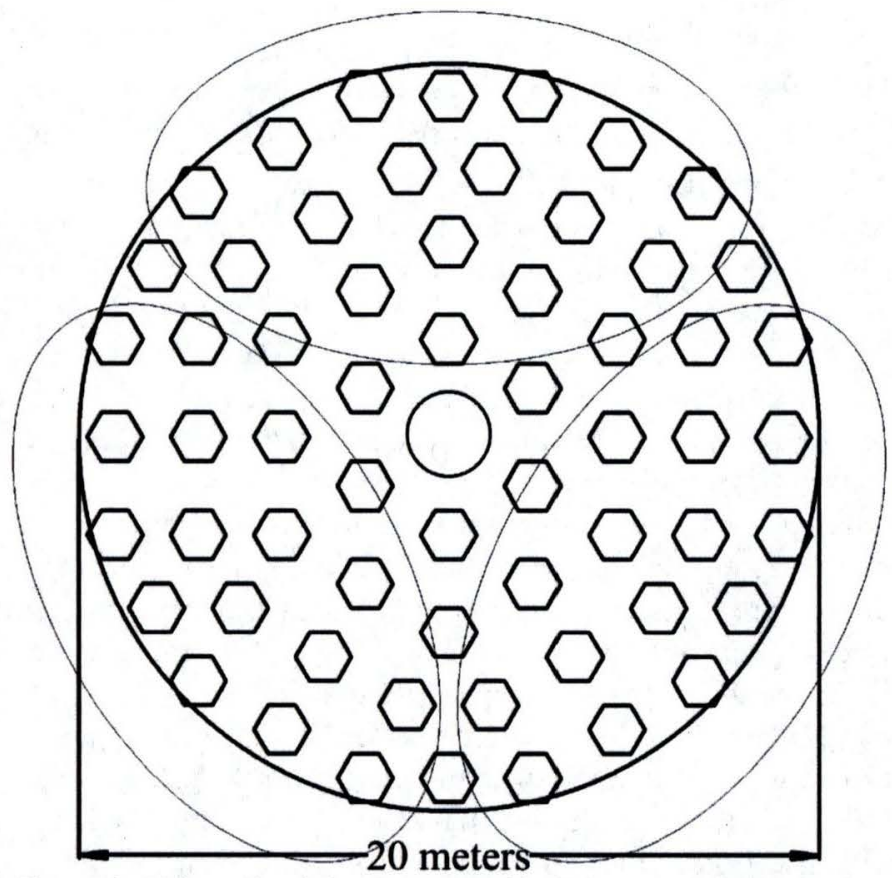

Figure 9. Using 60 of the James Webb Telescope mirrors in four concentric rings yields a 20 meter aperture diameter primary mirror. The light ellipses show the location of the drive coils.

Before further consideration of these high force and low force regimes, it is necessary to optimize coil performance with respect to available energy and weight. For most of the coils, the maximum coil diameter is fixed, for example, the mirror element coils cannot be much larger than the mirror elements themselves. So, optimizing the coils is equivalent to optimizing the total current flowing through them, given by $N I$ where $N$ is the number of wraps in the coil, and $I$ is the current. Letting $a$ be the radius of a coil and letting $l$ be the total length of wire in a coil, the total current can be rewritten as $l I /(2 \pi a)$. Recalling that the power, $W$, required to drive the coil is given by its power loss, $I^{2} R$, where $R$ is the coil resistance, the current can be replaced in the total current expression, yielding $l \sqrt{W / R} /(2 \pi a)$. Then, assuming that the majority of the resistance is from ohmic losses in the wire-as opposed to eddy currents and radiation - the resistance can be written in terms of the length, radius, $r$, and material resistivity, $\rho_{R}$, of the wire, yielding $\sqrt{W r^{2} l /\left(\pi \rho_{R}\right)} /(2 a)$. Then the total mass, $M$, of the coil can written in terms of the material mass density, $\rho_{m}$, times the volume of the wire yielding a final form for the total current,

$$
N I=\sqrt{W M /\left(\rho_{R} \rho_{m}\right)} /(2 \pi a)
$$

This is a surprisingly simple result, which is independent of the physical dimensions of the wire, leaving these parameters available for other design considerations.

As an example, if the mirror coils are each composed of one kilogram of aluminum $\left(\rho_{R}=2.8 \times 10^{-8} \Omega \mathrm{m}\right.$, $\left.\rho_{m}=2700 \mathrm{~kg} / \mathrm{m}^{3}\right), 1.3$ meters in diameter, and four watts of power is available to drive each coil, then the total current is $56 \mathrm{Amps}$ and the dipole moment is $75 \mathrm{~N}-\mathrm{m} / \mathrm{T}$. Now, recall that the force between two parallel wires, each carrying $56 \mathrm{Amps}$ and located about 2 meters apart is about 300 microNewtons/meter. The edges of the mirror elements are no more than 2 meters apart and each has three coils with 56 Amps of current in it, so the force between these coils, if they are operating at the same frequencies can be much greater than the 50 micro-Newtons needed to reorient the telescope. What this means is that the 60 primary mirror elements can be driven with the same three frequencies and can generate enough force to lock into a semi-rigid entity.

Now consider the drive coils. These large coils need to generate a sufficient magnetic field gradient to allow repositioning of the primary mirror elements. For the purposes of this example we will use the expression for the magnetic field along the centerline of a drive coil, as shown in Eqn. 4, to determine if a reasonable magnetic field gradient can be established. Taking the derivative of Eqn. 4 and using Eqn. 5 to replace the total current, $N I$, with values for the wattage and mass available for the drive coils yields

$$
\frac{d B}{d x}=-\frac{3 \mu_{0} N I a^{2} x}{2\left(a^{2}+x^{2}\right)^{5 / 2}}=-\frac{3 \mu_{0} a x \sqrt{W M /\left(\rho_{c} \rho_{m}\right)}}{4 \pi\left(a^{2}+x^{2}\right)^{5 / 2}} .
$$

If we assume that 100 Watts and 100 kilograms of aluminum are available for the energy and mass of each drive coil during a telescope reorientation, and if we assume the drive coils are 10 meters in radius then the total current from Eqn. 5 is 183 Amps. The magnetic field gradient at a distance of 5 meters along the drive coil axis is about $10^{-6} \mathrm{~T} / \mathrm{m}$, so the force on some of the primary mirror elements is about 75 microNewtons. Not every primary mirror element feels this force, some feel more and some less, but assuming the 60 mirror elements are locked together it should be sufficient to reorient the primary in under four hours.

The analysis above demonstrates that under relatively high wattage conditions the telescope can be reoriented in under four hours, but the mirror elements are all operating at the same three frequencies (one for each drive coil) and the resultant multi-body problem presents a complicated feedback and control problem-if one mirror element moves all the elements move to compensate. So now switch to a small force mode where the drive coils are each driven with 60 different frequencies at one watt each and the primary mirror elements each have their own three frequencies and are operating at only $1 / 10^{\text {th }}$ of a watt per frequency. Assuming the frequencies are spaced by a Hertz or two, each primary mirror element is now almost independently controlled from the others (when a primary mirror element moves it moves the drive coils a small amount, but assuming they are attached to the telescope submodule this is negligible).

One watt of power to a drive coil corresponds to $18 \mathrm{Amps}$ and $1 / 10^{\text {th }}$ watt in a mirror coil corresponds to 9 Amps and a 
dipole moment of $12 \mathrm{~N}-\mathrm{m} / \mathrm{T}$. Using Eqn. 6 and assuming that a primary mirror element might be 20 meters from one of the drive coils the magnetic field gradient is about $12 \mathrm{nT} / \mathrm{m}$ yielding a force of about 145 nano-Newtons-clearly sufficient to prevent gravity induced drifts. This demonstrates that the system can be placed into a low power mode with 60 Watts going to each drive coil and 18 Watts going into all of the primary mirror element coils.

While operating in this low power mode there is a significant benefit over micro-thrusters. The control forces can be finely adjusted through phase and amplitude changes to provide very small positional changes. Pico-Newton level force control should be easily achievable on time periods of less than 0.01 seconds. Consequently, for a $30 \mathrm{~kg}$ mass mirror element, acceleration adjustments into the $10^{-14} \mathrm{~m} / \mathrm{sec}^{2}$ range are achievable and can be changed quickly enough to limit positional errors to less than $10^{-18}$ meters, similar to that achieved with piezoelectric modulators and high end interferometers (of course this level of performance assumes the existence of a position monitoring system with similar or better specifications).

The necessary torques and corresponding angular positioning of the mirror elements is easily achieved within the above parameters. Assume a worst case where all of the 30 kilograms mass of a primary mirror element is on its outer edge (a 1.3 meter diameter hoop) so that its angular inertia is $13 \mathrm{~kg}-\mathrm{m}^{2}$. During the high wattage telescope reorientation process the magnetic field 20 meters from the drive coils is about 1 micro-Tesla, so the torque on the mirror coils can be as large as 75 micro-Newton-m. The angular acceleration is then about 6 micro-radians $/ \mathrm{sec}^{2}$, allowing rotation to any position within 30 minutes.

As shown in Figure 8, the secondary mirror is relatively far from the drive coils and we compensate for the drop in magnetic field gradient by making its coils larger, but depending on the telescope design details, this might not be adequate. It might be necessary to change the shape and extent of the drive coils to generate a larger magnetic field gradient at the secondary mirror. This issue, as well as many others, is beyond the scope of this paper. However, it has been demonstrated that a large aperture telescope composed of multiple free-floating primary mirror elements is feasibleadequate, yet finely controlled, forces can be obtained from a system with reasonable power and weight specificationsusing alternating magnetic field forces and frequency allocation.

\section{FAR FIELD POSSIBILITIES}

Our original hope was that a far field version of this satellite formation flying concept would prove feasible, one where two antennas located far from each other could be coupled together by substantial forces allowing a type of co-operative tractor beam effect to occur. However, analysis and experiment have shown that, even though relatively large repulsive forces can be created, only small attractive forces can be generated in such a far field configuration. Consider a pair of antennas facing each other, but located far apart. If one antenna launches an RF pulse containing total energy, $E_{1}$, then it will feel a recoil corresponding to the electromagnetic momentum it launched, $E_{1} / c$, where $c$ is the speed of light. When this pulse, composed of oscillating electric and magnetic fields, reaches the second antenna, synchronous currents in this second antenna can interact with the incoming pulse to produce a net force. However, the magnitude of this force is limited by conservation of momentum. Regardless of the details of the magnetic field/current interaction within the second antenna, its net final thrust is dictated by momentum conservation and is determined by what it does to the pulse arriving from the first antenna (reflection, absorption, or transmission) and the amount of additional electromagnetic momentum it launches. The bottom line is that the attractive forces generated by synchronously coupled antenna are limited to the sums of the powers emitted divided by the speed of light (repulsive forces can be higher because the RF pulses can recirculate between the two antennas pushing them apart [10]). So, for example, if 30 Watts can be emitted by each antenna, then the total attractive forces are limited $60 / c$ Newtons, where $c$ is the speed of light, i.e. 200 nanoNewtons. For most applications this is too much energy to expend to yield such a small force.

\section{CONCLUSION}

We have shown that by using alternating magnetic fields and synchronous currents that most of the satellite formation flying attributes listed in the introduction can be met.

a. This approach allows a wide dynamic range of forces allowing the satellite configuration to be reoriented or reconfigured within a few hours while still allowing very fine positioning through extremely fine force manipulation.

b. By operating at different frequencies each satellite can be independently positioned with minimal interaction with its neighbors.

c. No propellants are required and the weight of the coils and power requirements are within reasonable ranges. It may be feasible to further reduce the weight and power needed by using superconducting coils.

d. Finally, the proposed approach is applicable to short range systems (a few radii distances from the large drive coils), but it is not necessarily appropriate for far field operation.

\section{ACKNOWLEDGMENT}

We wish to thank Rick Birr for RF support and Robert Cox and Mike Csonka for aid in constructing test equipment. This work was supported by the NASA Office of the Chief Technologist. 


\section{REFERENCES}

1. Bristow, J., Folta, D., and Hartman, K., "A Formation Flying Technology Vision,” AIAA Space 2000

Conference and Exposiiton, Sept. 19-21, 2000, Long

Beach, Ca.

2. Scharf, D. P., Hadaegh, F. Y., and Ploen, S. R., "A Survey of Spacecraft Formation Flying Guidance and Control (Part II): Control," Proceeding of the 2004 American Control Conference, June-July 2004, Boston, Ma.

3. Ziemer, J. K., et. al. "Colloid Micro-Newton Thrusters for the Space Technology 7 Mission," 2010 IEEE Aerospace Conference, March 2010.

4. Tin Duc Le, et. al., "Orbital Formation Control of Multiple Spacecraft," 2010 American Control Conference. June-July 2010.

5. Rucai Che and Zhang, H. "Relative Orbit Design for Maintaining Constant Distance in Satellite Formation Flying," Conference on Computational Engineering In Systems Applications, October 2006, Beijing, China.

6. Sedwick, R. J. and Miller, D. W. , NIAC Phase II study, "Electromagnetic Formation Flight," http://www.niac.usra.edu/files/studies/final_report/838Se dwick.pdf ,August 2005.

7. Ahsun, U. and Miller, D. W., "Dynamics and Control of Electromagnetic Satellite Formations," Proceedings of the 2006 American Control Conference, June 2006, Minneapolis, Minn.

8. Sakai, S., et. al., "Electromagnetic formation flight for LEO satellites." Proc. Of $17^{\text {th }}$ workshop on JAXA Astrodynamics and Flight Mechanics, March 2008.

9. LaPointe, M. R., NIAC Phase I Study, "Formation Flying with Shepherd Satellites," http://www.niac.usra.edu/studies/607LaPointe.html Nov. 2001.

10. Bae, Young K., NIAC Phase I Study, "A ContaminationFree Ultrahigh Precision Formation Flight Method Based on Intracavity Photon Thrusters and Tethers," http://www.niac.usra.edu/studies/1374Bae.html March 2006.

11. Parlin, W.A., "The Torques and Forces between Short Cylindrical Coils Carrying Alternating Currents of Radio Frequency," Physics Review, Vol. 22, 1923, pp. 193-197.

12. Griffiths, D. J., Introduction to Electrodynamics, Third Edition. Prentice Hall, Upper Saddle River, New Jersey, 1999. See Section 6.1.2.

13. Wheeler, H.A., "Inductance Formulas for Circular and Square Coils," Proceedings of the IEEE, vol. 70, No. 12, Dec. 1982.

14. Lorrain, P., and Corson, D., "Electromagnetic Fields and Waves," Second Edition, W.H. Freeman and Co., San Francisco, 1970.

15. "Hubble Space Telescope," Wikipedia, The Free Encyclopedia. Wikimedia Foundation, Inc. July 2012. $<$ http://en.wikipedia.org/wiki/Hubble_Space_Telescope>

16. "The James Webb Space Telescope," NASA. July 2012. $<\underline{\text { http://www.jwst.nasa.gov/mirrors.html }>}$ 